\title{
Management of Cesarean Scar Pregnancy
}

\author{
${ }^{1}$ Dr. Shah Sapana R., ${ }^{2}$ Dr.Vyas Rupa C, ${ }^{3}$ Dr. Edwin Rebecca, ${ }^{4}$ Dr. Vasa Punit B, \\ ${ }^{5}$ Dr. Gamit Ankita F. \\ ${ }^{I}$ Associate professor and head of unitDepartment of Obstetrics and gynecologySmt. N.H.L. Municipal medical \\ college and Sheth V.S. General Hospital. \\ ${ }^{2}$ Assistant professor Department of Obstetrics and Gynecology Smt. N.H.L. Municipal medical college and \\ Sheth V.S. General Hospital \\ ${ }^{3,4,5}$ Resident doctorDepartment of Obstetrics and Gynecology Smt. N.H.L. Municipal medical college and Sheth \\ V.S. General Hospital
}

\begin{abstract}
To evaluate the diagnosis and treatment and long term follow up of cesarean scar pregnancy.
Methods: From January 2008 to December 2013, 12 cases of Cesarean scar pregnancy were diagnosed using transvaginal grey scale and color doppler ultrasound and managed by various approaches. Incidence, gestational age, ulrasound findings, serum beta-human chorionic gonadotropin ( beta-hCG) levels, flow profiles of transvaginal color doppler ultrasound, and different methods of treatment were recorded.

Results: The incidence of cesarean scar pregnancy was 1:2051. Gestational age at diagnosis ranged from 5 weeks +2 days to 10 weeks +4 weeks. The time interval from the last cesarean section to the diagnosis of Cesarean scar pregnancy ranged from 5 months to 8 years. Patients were treated by different methods as follows: transvaginal ultrasound-guided injection of methotrexate(MTX) into the gestational sac $(n=1)$, systemic methotrexate administration alone $(n=2)$, dilatation and curettage $(n=3)$, or local resection of the gestation mass $(n=6)$. After local or systemic MTX, high-velocity and low-impedance subtrophoblastic flow (resistance index $(R I)=0.35)$ persisted until beta-hCG returned to normal. One of 3 patients, treated by dilatation and curettage, underwent a hysterectomy because of uncontrolled profuse vaginal bleeding. The scar pregnancy mass took 2 months to 8 months for regression after treatment. None of the patient had uterine rupture.
\end{abstract}

Conclusion: Sonography guided methotrexate injection and/or systemic methotrexate are the treatment of choice to manage cesarean scar pregnancy less than 6-8 week's gestation without fetal heart activity. Dilatation and curettage leads to profuse vaginal bleeding and not recommended for cesarean scar pregnancy due to high morbidity. Those patients having persistent pain and recurrent bleeding, surgical resection for removal of mass and repair of defective site should be recommended.

Keywords: cesarean scar pregnancy, scar pregnancy, ectopic pregnancy.

\section{Introduction}

The implantation of a pregnancy within the previous cesarean scar is the rarest form of ectopic pregnancy $^{1}$. Cesarean scar pregnancy is defined as an ectopic pregnancy embedded in the myometrium of a previous cesarean scar ${ }^{1}$. A computer Medline and bibliography search has yielded only 112 cases reported from 1966 to $2005^{2}$. Jurkovic et al mentioned a series of 18 cesarean scar pregnancies, with a prevalence of 1 per 1800 pregnancies. $^{1,3}$

Early diagnosis with ultrasound helps to start appropriate treatment to preserve uterine integrity and future fertility without causing maternal complications ${ }^{4}$. Different methods of treatment include local and/or systemic administration of methotrexate, dilatation and curettage (D\&C), and local resection of the ectopic gestational mass. However, a delay in either diagnosis or treatment can lead to uterine rupture, hysterectomy, and significant maternal morbidity or even death. Although expectant and medical managements have been reported, termination of a cesarean scar pregnancy by laparotomy and hysterotomy, with repair of the accompanying uterine scar dehiscence, may be the best treatment option. ${ }^{1}$

We present our experience with 12 patients with cesarean scar pregnancy diagnosed using transvaginal color doppler ultrasound during a 6-year period and treated conservatively to preserve the uterus. Out of 12 , one woman required hysterectomy.

\section{Material And Methods}

From January 2008 to December 2013, total deliveries at our institute were 24,613. Out of them 9553 deliveries were through cesarean section. The cesarean section rate was $38.81 \%$. During this 6-year period, total of 240 ectopic pregnancies were diagnosed at our institute. Among these, 98 patients had history of previous cesarean section before the ectopic pregnancy; 12 of these were diagnosed as cesarean scar ectopic pregnancy 
using transvaginal ultrasound.

The diagnosis of cesarean scar pregnancy was confirmed if all of the following sonographic criteria ( Figure:1,2,3) were met $^{3,6}$ :

(1) the uterus was empty, with clearly demonstrated endometrium;

(2) the cervical canal was empty, without gestational sac or ballooning at the early diagnosis;

(3) presence of the gestation sac with or without a fetal pole, with or without fetal cardiac activity (depending on the gestation age) in the anterior part of the isthmic portion of the uterus; and an absence of normal myometrium between the urinary bladder wall and the gestational sac.

(4) color Doppler shows high velocity with low impedance peri-trophoblastic vascular flow clearly surrounding the sac.

There should not be free fluid in the pouch of Douglas, unless the cesarean scar pregnancy has ruptured.

After counseling about the risk of hysterectomy, all patients underwent conservative treatment including MTX injection, D\&C, or local resection of ectopic mass and suturing of lower segment by laparotomy or laparoscopy, because reproductive function was desired. The modality of treatment was as per the decision made by the individual surgeon. Informed consent was taken before treatment.

Local MTX injection under transvaginal ultrasound guidance was performed under general anesthesia with the patient in the lithotomy position. The gestational sac was punctured and aspirated under transvaginal sonographic guidance using a double-lumen ovum aspiration needle. Afterwards, $1 \mathrm{mg} / \mathrm{kg}$ methotrexate was injected slowly, resulting in a hyperechogenic area in the region of the former gestational sac. Daily serum $\beta$ hCG testing was performed during hospitalization and weekly thereafter 3 consecutive weeks, and after that two weekly until the level returned to $<5 \mathrm{mIU} / \mathrm{mL}$.

Transvaginal sonography using a Esaote MyLab 50 with a 5-MHz vaginal probe with color Doppler was carried out to monitor subtrophoblastic blood flow velocity. Vascular pattern and its waveform were observed and Doppler velocimetry (pulsatility index, resistance index and peak systolic velocities) were checked weekly until disappearance of subtrophoblastic blood flow. The size of the cesarean scar ectopic pregnancy mass was measured in centimeters till its persistance.

Two cases were given i.m MTX 1mg/kg. Follow-up with ultrasonography and color Doppler was done as mentioned above. Patients were advised abstinence until the resolution of the cesarean scar ectopic pregnancy.

Three cases underwent $\mathrm{D} \& \mathrm{C}$ due to misdiagnosis of missed abortion, since the the gestational sac was located in lower segment of the uterus.

Popping out of gestational sac through right side of previous scar was seen (Fig 4). Local resection, removal of ectopic mass and suturing of lower segment was carried out due to persistent pain and recurrent bleeding in six cases. Two cases were managed with laparoscopy, and in remaining four laparotomy was carried out.

\section{Results}

Various clinical data are presented in Table 1 and 2.

The mean age of the patients was 27.4 (range, 20-35) years. Gestational age at diagnosis ranged from 5 weeks +2 days to 10 weeks +4 weeks. The time interval from the last Cesarean section to the diagnosis of Cesarean scar pregnancy ranged from 5 months to 8 years. Fetal cardiac activity was present in six cases. .

The residual ectopic cesarean scar persisted in-situ for several months after conservative treatment with MTX even though $\beta$-hCG had declined to a normal limit The scar pregnancy mass took 2 months to 8 months time for regression after treatment. None of the patient had uterine rupture.

Three cases underwent D\&C due to misdiagnosis of missed abortion, since the the gestational sac was located in lower segment of the uterus. As the gestational sac was embedded in scar of previous cesarean section, profuse hemorrhage occurred in all 3 cases. Uncontrolled bleeding of about $700 \mathrm{ml}$ occurred in case no. 3 required hysterectomy. Histopathology report demonstrated presence of abundant chorionic villi and trophoblastic tissue inside the fibrotic myometrium. Other 2 cases had also profuse bleeding of about $550 \mathrm{ml}$, managed with Foleys' catheter balloon instilled with $50 \mathrm{ml}$ of normal saline, and it acted as a successful temponade. All three women required 3 units of PCV.

In six cases, local resection, removal of ectopic mass and suturing of lower segment was carried out due to persistent pain and recurrent bleeding. Two out of six cases were managed with laparoscopy, and in remaining four laparotomy was carried out. Urinary bladder was adherent to scar tissue, dissected carefully in all six cases. Three of them required bilateral uterine artery ligation by O'leary technique for control of severe haemorrhage and required 2 units of PCV. Histopathology report showed the presence of the dense interstitial fibrosis of myometrium adjacent to the chorionic vill. 


\section{Discussion}

In 1978, Larsen and Solomon reported the first case of cesarean scar pregnancy. ${ }^{1}$ Present study shows the incidence of cesarean scar pregnancy is $1: 2051$, which coincides with Jurkovic et al ${ }^{1,3}$ series in which prevalence is 1 per 1800 pregnancies.

The exact etiology of cesarean scar pregnancy is unknown. There are several hypotheses, proposed by different authors. Vial et $\mathrm{al}^{5}$ found two different type of cesarean scar pregnancy. First type of cesarean scar pregnancy is an implantation of conceptus on prior cesarean scar and it grows towards the cervicoisthmic space or the uterine cavity. This type of cesarean scar pregnancy may grow up to term but with the risk of torrential and life threatening haemorrhage. Second type of cesarean scar pregnancy is a deep implantation into a cesarean scar defect, and it grows towards the urinary bladder and abdominal cavity. Such cesarean scar pregnancy is more prone to rupture. Other hypothesis argues that the conceptus enters the myometrium through a microscopic dehiscent tract or defect in the cesarean section scar. ${ }^{6}$

Seow et al reported the first case of cesarean scar pregnancy following in vitro fertilization-embryo transfer in 2000 . He also recommended that, the embryo should be transferred at least $4 \mathrm{~cm}$ from the cervix to avoid the caesarean scar and cervical pregnancy, in patients with a history of a cesarean section. ${ }^{4}$

Transvaginal ultrasonography with color Doppler is very useful for diagnosis of cesarean scar pregnancies. It must be distinguished from other types of abnormally implanted pregnancies, including cervical, cervicoisthmic, and cervicoisthmic corporeal pregnancies, as outcome and treatment may differ in each. Cesarean scar pregnancy is different from intrauterine pregnancy with morbidly adherent placenta in that it occurs in the first trimester, involves the complete embedding of the gestational sac in the myometrium and it is more aggressive. The uterine myometrium between the urinary bladder and the gestational sac becomes very thin or disappears due to enlargement of the sac. The thin, serosal layer is present. Robert et $\mathrm{al}^{9}$ mentioned "salmon red" appearance of cesarean scar pregnancy under a laparoscope. With advent of transvaginal sonography with color Doppler and MRI, it has been possible to diagnose it earlier in the gestation, and to adopt more conservative approach, as treatment.

Few reports of cesarean scar pregnancies successfully treated conservatively with MTX and expectant management are available. ${ }^{4-8}$ Several protocols: systemic single-dose MTX or multiple-dose MTX with alternate-day folinic acid rescue or local MTX injection into the sac are reported. MTX resulted in resolution of cesarean scar pregnancy without surgical intervention. Godin et al reported a case of transvaginal injection of potassium chloride into the fetal heart and MTX to the gestational sac and surrounding myometrium in a 9week viable cesarean scar pregnancy. Cesarean section scar ectopic mass resolved completely, but hysterosalpingography showed dehiscence of the uterine scar 16 weeks later. ${ }^{6}$ In some cases, hemorrhage occurred after MTX treatment, requiring emergency laparotomy. ${ }^{1,5,7}$ Lai et al reported a case in which, two weeks after transvaginal sonography-guided local injection of MTX into an gestational sac embedded into scar, an emergency laparotomy was required due to onset of profuse vaginal bleeding from the ruptured uterine scar. Haimov-Kochman R et al recommended that MTX delivery and expectant management may be a safe treatment alternative in cesarean scar pregnancy less than $6-8$ week's gestation without fetal heart activity. ${ }^{8}$

Some authors propose that $\mathrm{D} \& \mathrm{C}$ should not be first-line therapy due to the risk of perforation and catastrophic hemorrhage ${ }^{10}$ Present study also reported profuse vaginal bleeding during D\&C in all three cases of cesarean section pregnancy, one of them required hysterectomy. Lee et al described laparoscopic resection of a cesarean section ectopic pregnancy. ${ }^{10}$ Graesslin et al described successful management of cesarean section ectopic pregnancy with the use of systemic methotrexate followed by D\&C. ${ }^{11}$ Uterine artery embolization to reduce hemorrhage has also been described as adjunctive therapy. ${ }^{12}$

Treatment options like systemic and local MTX, local potassium chloride, eventhough successful could be expected to leave the uterine scar defect that will accompany repeat cesarean scar implantation and may rupture in future pregnancy. Surgical resection offers the opportunity to remove the ectopic pregnancy, as well as repairs the defect simultaneously and should be recommended as soon as the diagnosis is confirmed.

Table 1: Clinical data of patients with cesarean scar pregnancy

\begin{tabular}{|c|c|c|c|c|c|}
\hline Case & $\begin{array}{c}\text { Age } \\
\text { (Years) }\end{array}$ & $\begin{array}{c}\text { Time interval } \\
\text { from last CS } \\
\text { (Years) }\end{array}$ & $\begin{array}{c}\text { Gestational } \\
\text { Age at diagnosis } \\
\text { (weeks) }\end{array}$ & $\begin{array}{c}\text { Fetal heart activity } \\
\text { Yes/No }\end{array}$ & $\begin{array}{c}\text { Initial } \beta \text {-hCG } \\
\text { level (mIU/mL) }\end{array}$ \\
\hline 1 & 22 & 5 & 5 weeks +3 days & No & 5123 \\
\hline 2 & 25 & 4 & 8 weeks +6 days & Yes & 13420 \\
\hline 3 & 20 & 1 & 8 weeks +1 days & No & 7239 \\
\hline
\end{tabular}


Management of Cesarean Scar Pregnancy

\begin{tabular}{|c|c|c|c|c|c|}
\hline 4 & 35 & $8 \& 5$ & 8 weeks +6 days & No & 6082 \\
\hline 5 & 23 & 1 & 7 weeks +3 days & No & 9325 \\
\hline 6 & 27 & 6 & 8 weeks +6 days & No & 5600 \\
\hline 7 & 28 & 5 & 8 weeks +6 days & No & 9300 \\
\hline 8 & 32 & $4 \& 2$ & 9 weeks +2 days & Yes & 13676 \\
\hline 9 & 26 & 0.5 & 9 weeks +4 days & Yes & 16077 \\
\hline 10 & 26 & 3 & 10 weeks +2 days & Yes & 18790 \\
\hline 11 & 30 & 8 & 8 weeks +3 days & Yes & 20949 \\
\hline 12 & 35 & $7 \& 5$ & 8 weeks +5 days & Yes & 23780 \\
\hline
\end{tabular}

Table 2: Various methods of treatment and follow-up of cesarean scar pregnancy.

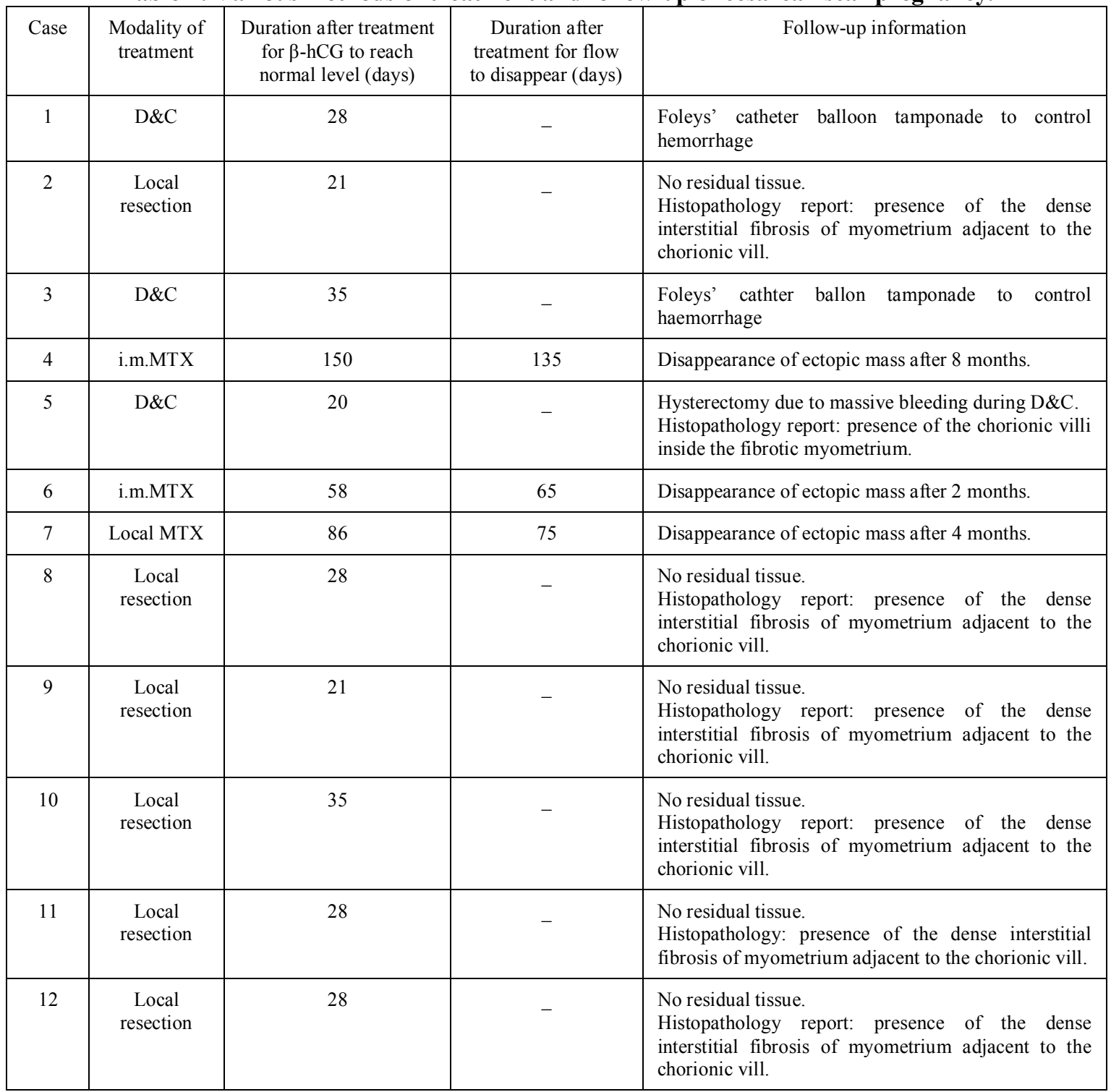


Fig 1: Transvaginal ultrasonography showed empty uterine cavity and cervical canal, location of gestational $\mathrm{sac}(\mathrm{GS})$ in anterior uterine wall at the level of isthmus embedded in the previous scar.(Longitudinal section)

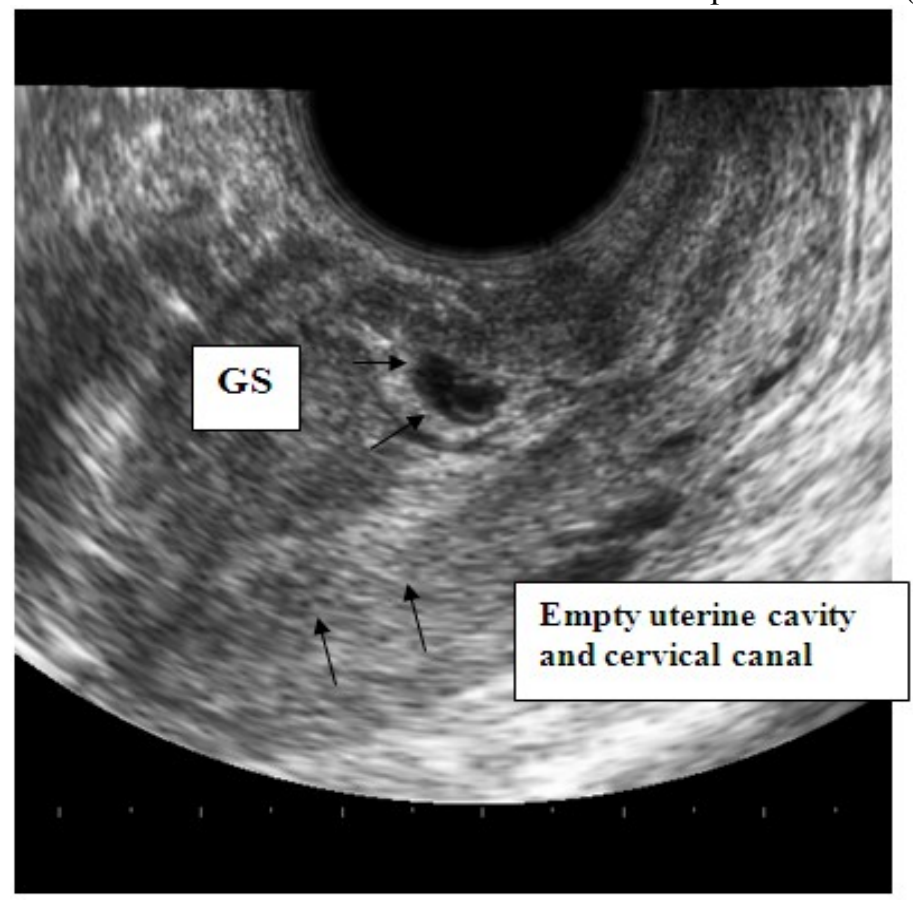

Fig 2: Transvaginal ultrasonography showed empty uterine cavity and cervical canal, location of gestational sac (GS) in anterior uterine wall at the level of isthmus embedded in right side of previous scar. (Transverse section)

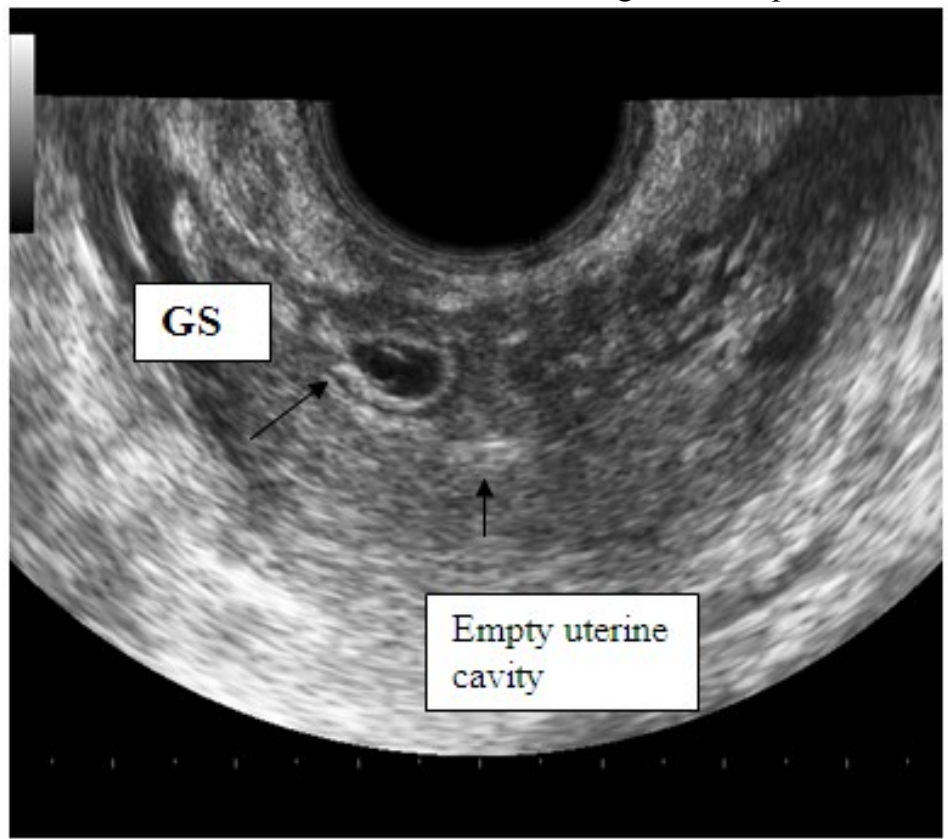


Fig 3: Color Doppler shows peri-trophoblastic vascular flow clearly surrounding the sac

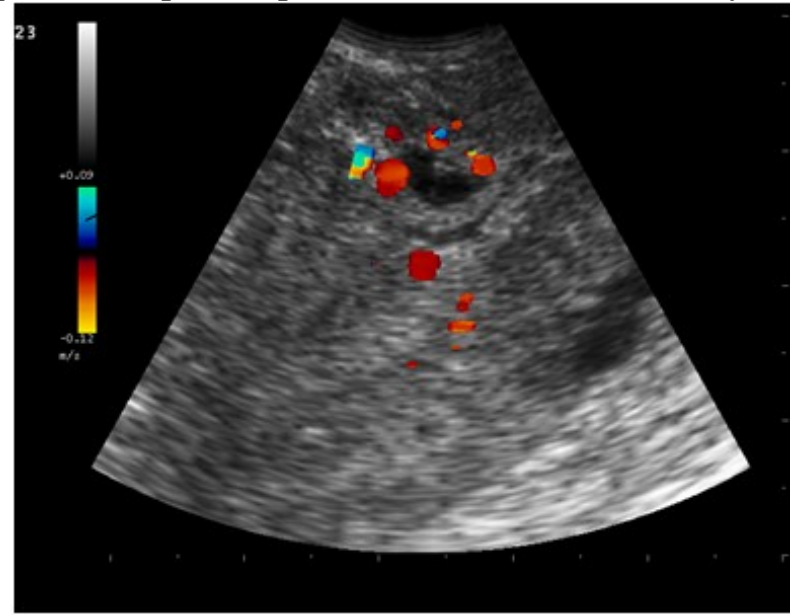

Fig 4. Popping out of gestational sac through right side of previous scar.

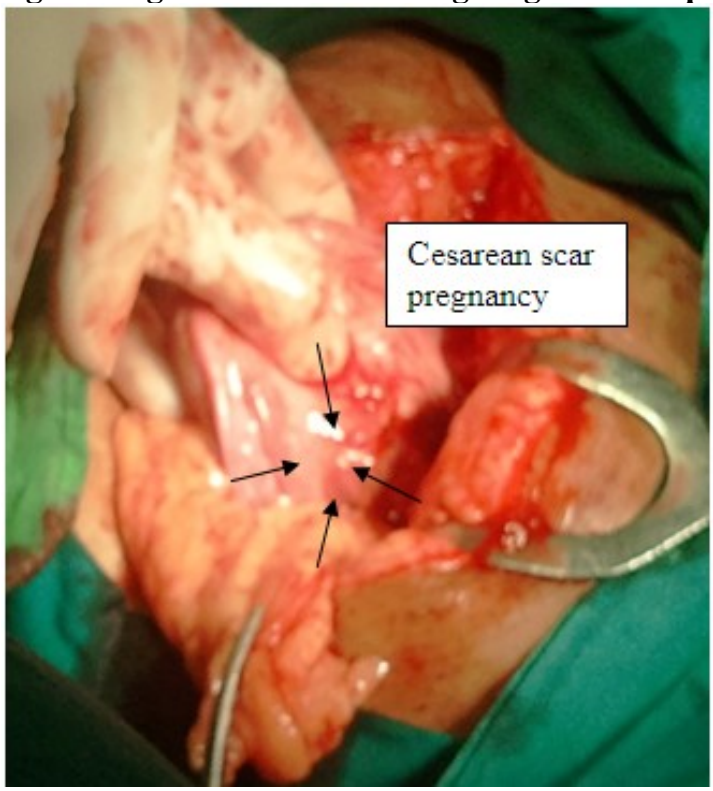

\section{References}

[1]. Fylstra DL, Pound-Chang T, Miller MG, et al. Ectopic pregnancy within a cesarean delivery scar: a case report. Am J Obstet Gynecol 2002;187:302-4.

[2]. Elizabith A, Baher M, Mindy M, et al. Caesarean delivery scar ectopic pregnancy. A case report. Ultrasound Quarterly 2010;26:106-8.

[3]. Jurkovic D, Hillaby K, Woelfer B,et al. First trimester diagnosis and management of pregnancies implanted in the lower uterine Caesarean section scar. Ultrasound Obstet GynecoL 2003;21:220-7.

[4]. Seow KM, Cheng WC, Chuang J, et al. Methotrexate for cesarean scar pregnancy after in vitro fertilization and embryo transfer: a case report. J Reprod Med 2000; 45:754-7.

[5]. C. M. Yan, “A report of four cases of caesarean scar pregnancy in a period of 12 months," Hong Kong Medical Journal 2007;13:141-3.

[6]. Godin PA, Bassil S, Donnez J. An ectopic pregnancy developing in a previous caesarian section scar. Fertility and Sterility 1997;67:398-400.

[7]. Lai YM, Lee JD, Lee CL, et al. An ectopic pregnancy embedded in the myometrium of a previous cesarean section scar. Acta Obstet Gynecol Scand 1995;74:573-6.

[8]. Haimov-Kochman R, Sciaky-Tamir Y, Yanai N, et al. Conservative management of two ectopic pregnancies implanted in previous uterine scars. Ultrasound Obstet Gynecol 2002;19:616-9.

[9]. Roberts H, Kohlenber C, Lanzarone V, et al. Ectopic pregnancy in lower segment uterine scar. Aust NZ J Obstet Gynecol 1998; 38:114-6.

[10]. Lee CL, Wang CJ, Chao A, et al. Laparoscopic management of an ectopic pregnancy in a previous Caesarean section scar.Hum Reprod 1999; 14(5):1234-6.

[11]. Graesslin O, Dedecker F, Quereux C, et al. Conservative treatment of ectopic pregnancy in a cesarean scar Obstet Gynecol 2005; 105(4):869-871

[12]. Badawy SZ, Etman A, Singh M, et al. Uterine artery embolization: the role in obstetric and gynecology. Clin Imaging 2001; 25:288-95. 